\title{
Effect of microbeam geometry on the nano-mass sensor performance
}

\author{
Jahanbakhsh Reisi Ardali, Reza Ghaderi*, and Farhad Raeiszadeh \\ Department of Mechanical Engineering, Faculty of Technical and Engineering, Shahrekord Branch, Islamic Azad University, \\ Shahrekord, Iran
}

Received: 14 February 2018 / Accepted: 24 February 2019

\begin{abstract}
Microbeams have a wide range of applications as sensors and actuators in nanotechnology, biotechnology, microelectromechanical systems, and optics. Given their micrometer dimensions, these beams make precision mass sensors of sub-nanogram accuracy. An important challenge regarding mass sensors is to enhance their sensitivity and accuracy. Considering the fact that, this type of sensor operates based on the resonance frequency variations caused by nanoparticle absorption in the dynamic mode, the geometry of the microbeam is considered an important parameter affecting their sensitivity. This paper studies the rectangular microbeam, which is one of the most commonly used types of mass sensors. Three main models were selected by applying inner and outer cuts on the microbeam, and vibrating simulation was carried out using ABAQUS software for a total of 36 mass sensor configurations with different aspect ratios. Simulation results in two selected rectangular microbeam models with outer cuts show the sensitivity of the microsensor increases with increased microbeam rigidity. The triangular hollow microbeam was found to be the best design among the four models selected to be used as mass sensors.
\end{abstract}

Keywords: Mass microsensor / microbeam / frequency sensitivity / inner and outer cut

\section{Introduction}

Microbeam structures are extensively used in various sensors and actuators in electromagnetics, optics, and biology [1-3]. An important application of microbeams is in mass nanosensors. Given the nanoscale geometric dimensions of this type of beams, their accuracy as a mass sensor is at nanogram. Improving the design of the microsensor structure is critical to have a higher accuracy and sensitivity. Before 1990, microbeams were rarely used in microsensor applications. Mazzola and Foder [4] used atomic force microscopy to detect protein substrate. Later, Dammers et al. [5] measured specific antigen-antibody interactions by detecting adhesion force using microbeam bending. Today, microbeams are generally used to load the target on the surface and to determine the change in the resonance frequency after mass loading [6-8]. The resonance frequency of microbeams decreases with mass and is positively correlated with the specific binding of antigen and antibody $[9,10]$. Goericke and King [11] studied the microsensors response to surface stress. They found that microsensors sensitivity increases by decreasing the length to width ratio.

\footnotetext{
* e-mail: reza.ghaderi@ymail.com
}

Loui et al. [12] designed five different types of piezoelectric microbeams with different geometric shapes and concluded that microbeams with smaller geometric ratio are more suitable for the microsensor applications. These findings show that microbeams with smaller geometric ratio have higher rigidity and are more suitable for surface loading. Experimental results show a smaller deflection in wide and short microbeams under surface loading. Salehi Khojin et al. [13] studied the vibrating behavior of piezoelectric microbeam as a mass sensor in the air environment. They used the modal analysis method to solve the differential equation of motion of a piezoelectric microbeam with an added mass at one of its ends. Xie et al. [14] proposed a dynamic method for the application of rectangular microbeams as mass sensors using the Rayleigh-Ritz method. Faegh et al. [15,16] proposed a self-measuring biosensor using a self-measuring electrical circuit for operation in a fluid environment.

Since the geometric shape of microbeams significantly affects the sensitivity of mass microsensors, this paper addresses the effect of microbeam geometry on the frequency sensitivity of the microsensors. The inner or outer cuts that form in the microbeams change the sensitivity of the sensor by changing its rigidity; therefore, in addition to the geometric shape, the cut is also studied as an important factor in this paper. 


\section{Dynamic modeling of microbeams as mass sensors}

Based on the continuous beam model and using the EulerBernoulli theory and the Lagrangian method, the governing differential equation motion of a beam can be expressed as follows:

$$
\rho A(x) \ddot{v}+\left[E I(x) v^{\prime \prime}\right]^{\prime \prime}+C \dot{v}=0,
$$

here, $\rho, A, E$, and $I$ are density, cross-sectional area, elastic modulus, and moment of inertia of the surface, respectively. $C$ represents the damping of the system. Using the variable separation method and the Galerkin linear approximation, the deformation of each point on microbeam can be expressed as:

$$
v=\sum_{i=1}^{n} C_{n} \phi_{n}(x) e^{-i \omega_{n} t},
$$

where $\phi_{n}, C_{n}$ and $\omega_{n}$ represent the mode shape, the modal range, and the natural frequency, respectively. For a simply supported beam, $\phi_{n}$ is:

$$
\begin{aligned}
\phi_{n}(x) & =A_{n}\left(\cos \alpha_{n} x-\cosh \alpha_{n} x\right)+B_{n}\left(\sin \alpha_{n} x\right. \\
& \left.-\sinh \alpha_{n} x\right),
\end{aligned}
$$

where, $\alpha_{n}$, which represents the modal wavenumbers, is obtained from the following equation:

$$
\cos \left(\alpha_{n} L\right) \cosh \left(\alpha_{n} L\right)+1=0
$$

If the mass absorbed by the microbeam is much smaller than the microbeam mass, the change in the beam mode shape will be negligible. Therefore, the resonance frequency of the microbeam after absorbing the nanoparticle can be obtained using the energy method and the Rayleigh-Ritz theory. According to the Rayleigh-Ritz theory, the average kinetic energy $(T)$ and the average potential energy $(U)$ are equal at the resonance frequency. Therefore, when a particle with the mass $m_{e}$ is added to the microbeam, the sum of kinetic energy of the particle and the microbeam is equal to the potential strain energy of the microbeam:

$$
U=T+T_{\text {particle }}
$$

The kinetic energy of the microbeam and the kinetic energy of the nanoparticle at any resonance frequency can be expressed as:

$$
\begin{gathered}
T=\frac{1}{2} \int_{V} \rho A \dot{v}^{2} d V=\frac{1}{2} \rho A{\omega^{\prime}}_{n}^{2} C_{n}^{2}, \\
T_{\text {particle }}=\frac{1}{2} m_{e} \omega_{n}^{\prime 2} C_{n}^{2} \phi_{n}^{2}(L),
\end{gathered}
$$

here, $\omega_{n}$ is the resonance frequency of microbeam after absorbing the nanoparticle. Since the mode shape of the microbeam does not change after absorbing the nanoparticle, the strain energy of the microbeam can be approximately equated to the kinetic energy of the microbeam before the nanoparticle is absorbed:

$$
U=\frac{1}{2} \rho A \omega_{n}^{2} C_{n}^{2}
$$

Thus, using the Rayleigh-Ritz method and according to equations (1-8), the resonance frequency of microbeam after absorbing the nanoparticle can be expressed as:

$$
\frac{\omega_{n}^{\prime}}{\omega_{n}}=\frac{1}{\sqrt{1+\left(m_{e} / \rho A\right) \phi_{n}^{2}(L)}} .
$$

Equation (9) can be used for uniform rectangular microbeams with an acceptable accuracy. However, if the microbeam has a geometric discontinuity, this equation will not yield suitable results. Therefore, the finite element method is considered the best method for this type of beams. Since this paper examines the microbeams with geometric discontinuity, ABAQUS software was used for the vibrating analysis of the microbeams using the finite element method.

\section{Different cuts in rectangular and triangular microbeams}

Owing to their microscale dimensions, microbeams are considered an ideal option for mass nanosensors. In this type of sensor, measurements are made by vibrating actuation of the microbeam at its flexural resonance frequency. The resonance frequency changes by absorbing the nanoparticle and the changes in the vibrating motion of the microbeam become evident as the vibrating amplitude decreases. Certainly, the higher the microbeam sensitivity is to absorbing the nanoparticle, the higher is the accuracy and performance of the sensor. The geometric shape of microbeams is a factor that affects their sensitivity as a mass nanosensor. Therefore, design and selection of suitable geometry for the beam that can optimize the microbeam sensitivity to nanoparticle absorption is a challenge in mass nanosensor design. Not only the geometry but also the inner and outer cuts formed in the microbeam affect its mass sensitivity. Four general models (Fig. 1) were selected to investigate the impacts of the cuts formed in the microbeam and the microbeam aspect ratio. The four models were created with inner or outer cuts of the rectangular and triangular beams.

Different aspect ratios were considered for each of the four models according to Tables 1-4 to investigate the impact of inner and outer cuts on frequency variations in microbeams.

\section{Simulation of vibrating motion of microbeam as nano-mass sensor}

Due to their geometric discontinuities, the dynamic modeling of the vibrating motion of microbeams in Figure 1 is not only difficult using the beam theories but also does 


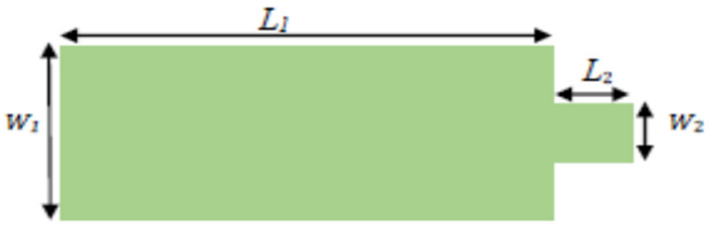

Model (A)

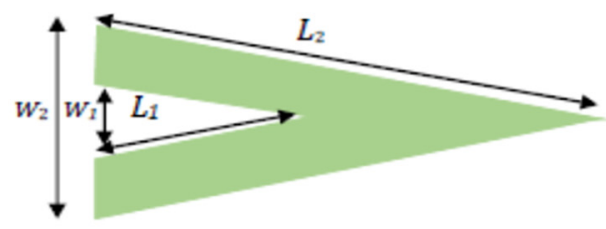

Model (C)

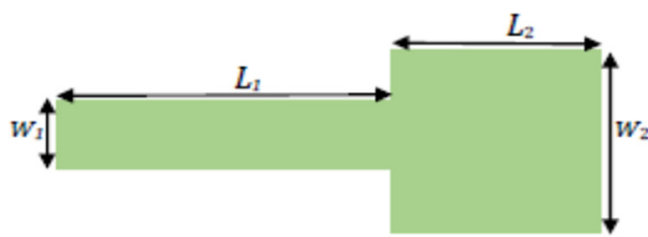

Model (B)

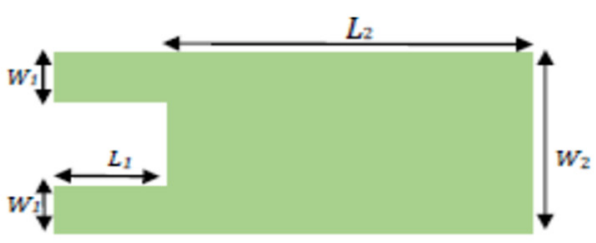

Model (D)

Fig. 1. Inner and outer cuts in rectangular and triangular microbeams.

Table 1. Different configurations of the microbeam model (A).

\begin{tabular}{llll}
\hline Model $(\mathrm{A})$ & $\mathrm{L}_{1} / \mathrm{L}_{2}=0.5$ & $\mathrm{~L}_{1} / \mathrm{L}_{2}=1$ & $\mathrm{~L}_{1} / \mathrm{L}_{2}=2$ \\
\hline $\mathrm{W}_{1} / \mathrm{W}_{2}=1 / 3$ & $\mathrm{~A}_{11}$ & $\mathrm{~A}_{12}$ & $\mathrm{~A}_{13}$ \\
$\mathrm{~W}_{1} / \mathrm{W}_{2}=1 / 2$ & $\mathrm{~A}_{21}$ & $\mathrm{~A}_{22}$ & $\mathrm{~A}_{23}$ \\
$\mathrm{~W}_{1} / \mathrm{W}_{2}=2 / 3$ & $\mathrm{~A}_{31}$ & $\mathrm{~A}_{32}$ & $\mathrm{~A}_{33}$ \\
\hline
\end{tabular}

Table 2. Different configurations of the microbeam model (B).

\begin{tabular}{llll}
\hline Model (B) & $\mathrm{L}_{2} / \mathrm{L}_{1}=1 / 3$ & $\mathrm{~L}_{2} / \mathrm{L}_{1}=1 / 2$ & $\mathrm{~L}_{2} / \mathrm{L}_{1}=1$ \\
\hline $\mathrm{W}_{1} / \mathrm{W}_{2}=1 / 3$ & $\mathrm{~B}_{11}$ & $\mathrm{~B}_{12}$ & $\mathrm{~B}_{13}$ \\
$\mathrm{~W}_{1} / \mathrm{W}_{2}=1 / 2$ & $\mathrm{~B}_{21}$ & $\mathrm{~B}_{22}$ & $\mathrm{~B}_{23}$ \\
$\mathrm{~W}_{1} / \mathrm{W}_{2}=2 / 3$ & $\mathrm{~B}_{31}$ & $\mathrm{~B}_{32}$ & $\mathrm{~B}_{33}$ \\
\hline
\end{tabular}

Table 3. Different configurations of the microbeam model (C).

\begin{tabular}{llll}
\hline Model $(\mathrm{C})$ & $\mathrm{L}_{1} / \mathrm{L}_{2}=1 / 3$ & $\mathrm{~L}_{1} / \mathrm{L}_{2}=1 / 2$ & $\mathrm{~L}_{1} / \mathrm{L}_{2}=2 / 3$ \\
\hline $\mathrm{W}_{1} / \mathrm{W}_{2}=1 / 3$ & $\mathrm{C}_{11}$ & $\mathrm{C}_{12}$ & $\mathrm{C}_{13}$ \\
$\mathrm{~W}_{1} / \mathrm{W}_{2}=1 / 2$ & $\mathrm{C}_{21}$ & $\mathrm{C}_{22}$ & $\mathrm{C}_{23}$ \\
$\mathrm{~W}_{1} / \mathrm{W}_{2}=2 / 3$ & $\mathrm{C}_{31}$ & $\mathrm{C}_{32}$ & $\mathrm{C}_{33}$ \\
\hline
\end{tabular}

not provide accurate results. Therefore, the finite element method is considered a suitable method for analyzing the vibrating motion of microbeams. ABAQUS software was used to simulate the microbeam vibrating motion using the finite element method. After drawing the geometry of the microbeams, the twenty-node hexahedral element was selected for meshing. The optimal number of elements was determined for each model and their aspect ratios by performing a mesh study, and the mesh size was reduced
Table 4. Different configurations of the microbeam model (D).

\begin{tabular}{llll}
\hline Model $(\mathrm{D})$ & $\mathrm{L}_{1} / \mathrm{L}_{2}=1 / 3$ & $\mathrm{~L}_{1} / \mathrm{L}_{2}=1 / 2$ & $\mathrm{~L}_{1} / \mathrm{L}_{2}=2 / 3$ \\
\hline $\mathrm{W}_{1} / \mathrm{W}_{2}=1 / 3$ & $\mathrm{D}_{11}$ & $\mathrm{D}_{12}$ & $\mathrm{D}_{13}$ \\
$\mathrm{~W}_{1} / \mathrm{W}_{2}=1 / 2$ & $\mathrm{D}_{21}$ & $\mathrm{D}_{22}$ & $\mathrm{D}_{23}$ \\
$\mathrm{~W}_{1} / \mathrm{W}_{2}=2 / 3$ & $\mathrm{D}_{31}$ & $\mathrm{D}_{32}$ & $\mathrm{D}_{33}$ \\
\hline
\end{tabular}

Table 5. Simulation parameters for piezoelectric MC [15].

\begin{tabular}{llll}
\hline & Material & $\mathrm{E}(\mathrm{GPa})$ & $\rho\left(\mathrm{kg} / \mathrm{m}^{3}\right)$ \\
\hline Main layer & $\mathrm{Si}$ & 180 & 2330 \\
Piezo layer & $\mathrm{ZnO}$ & 78 & 6390 \\
\hline
\end{tabular}

until the natural frequency value or the vibrational altitude of the elements converged to a fixed value. Considering that each model has a different geometry and that each model is studied at different aspect ratios, the mesh size or the number of selected elements is different in different cases. Given that the twenty-node hexahedral element was selected, the optimal mesh size was found to be between 14000 to 23000 . The microsensor is considered a simply-supported beam whose free end absorbs the nanoparticle on the longitudinal symmetry axis of the microbeam. The nanoparticle was constructed using a sphere attached to the microbeam. Physical properties of these microbeams, which was used in simulation, are given in Table 5.

In mass sensors, the resonance frequency is considered the most important quantity in vibrating motion since the extent to which the nanoparticle is absorbed can be measured using the changes in the resonance frequency. Considering the limitations of biological samples and the necessity of microbeam functionality in a liquid environment, vibration of the beam is always influenced 


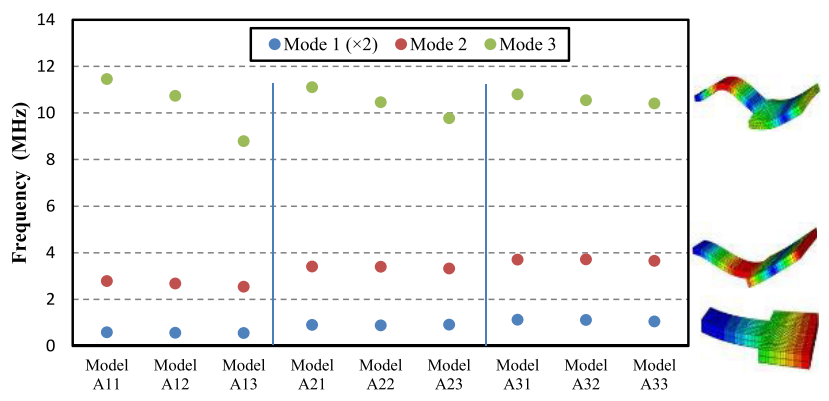

(a)

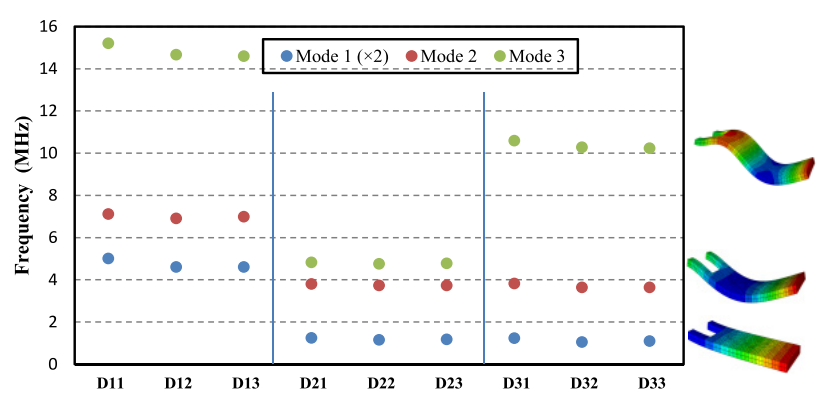

(c)

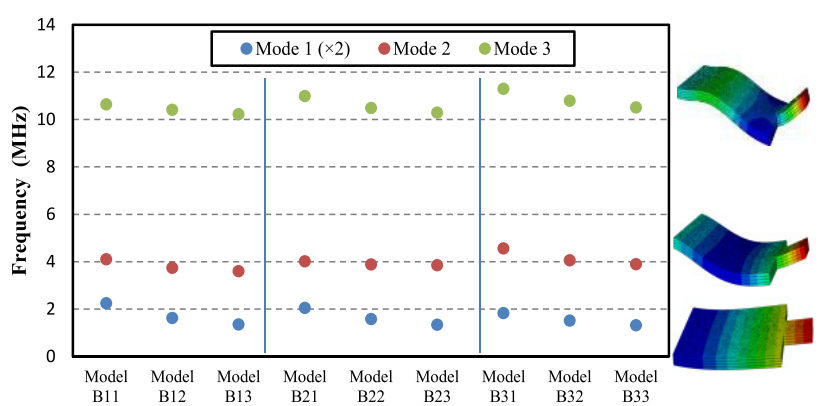

(b)

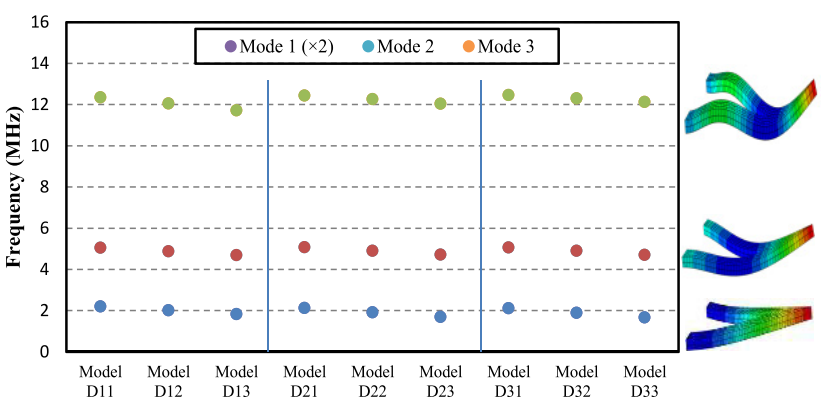

(d)

Fig. 2. Resonance frequency of the first three vibrating modes of the selected microbeams.
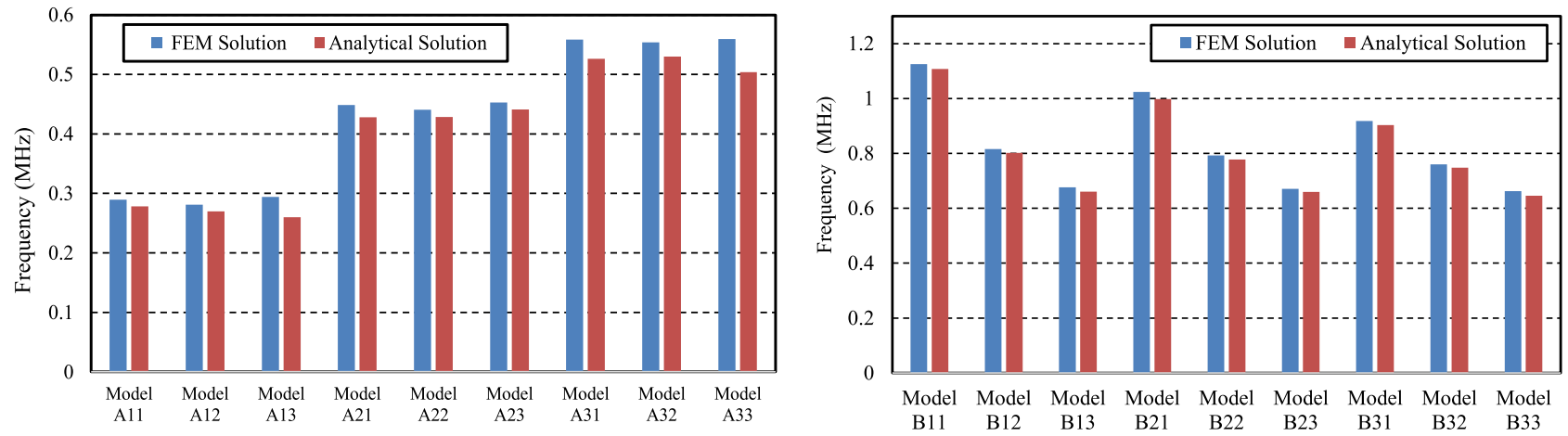

Fig. 3. Comparison between the FEM and analytical results for the natural frequency.

by unwanted flow-induced and thermal-induced noise. The microbeam should have a high resonance frequency to minimize such noises and reduce their undesirable effects on microbeam vibration. Therefore, microbeams with a higher resonance frequency are more accurate and perform better as mass sensors. Figure 2 shows the resonance frequency of the first three vibration modes of the selected microbeams in different configurations. Results in Figure $2 \mathrm{a}$ show that, by increasing the length of the narrower part of the microbeam and, thereby, reducing its stiffness, the resonance frequency decreases in the first three modes. Higher resonance frequencies are more favorable in mass sensors. Therefore, it is necessary to minimize the length of the narrower part of the beam. The microbeam in the model (B) is the opposite of the model (A). In this type of microbeam, by reducing the length of the narrower part and, thereby, increasing the microbeam stiffness, the resonance frequency increases. Figures $2 \mathrm{c}$ and $2 \mathrm{~d}$ show that increased length of the omitted part decreases the resonance frequency.

As it was mentioned earlier, discontinuities in the geometry of the microbeams make it difficult to calculate the natural frequencies. Considering the geometry of microbeams Type A and B that feature less discontinuity, the first mode of their natural frequencies was calculated and studied for aspect ratios selected in Tables 1 and 2 by FEM and analytical methods. Figure 3 compares the FEM and analytical results for the natural frequency. It is evident that FEM and analytical results for Types A and B are in agreement for all selected aspect ratios.

Nine different aspect ratios were considered according to Tables 1-4 to examine the effect of aspect ratios on frequency sensitivity in the three selected models. This analysis aimed to select an optimal dimensional ratio for 


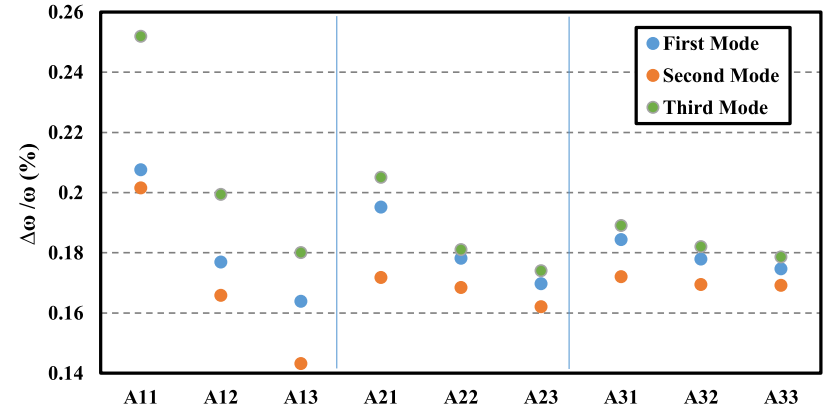

(a)

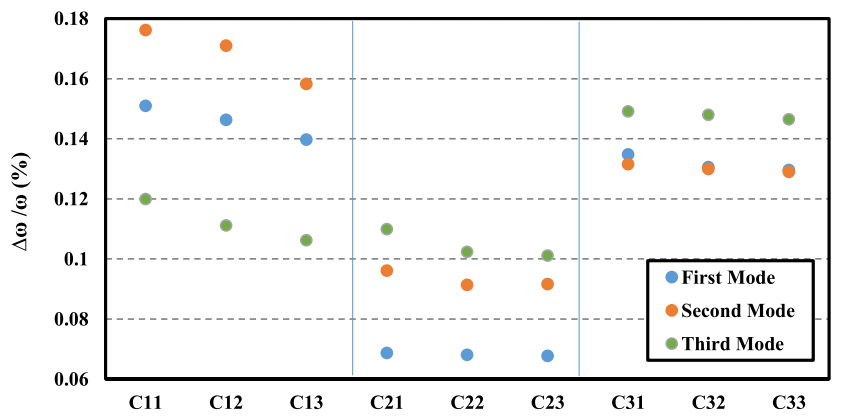

(c)

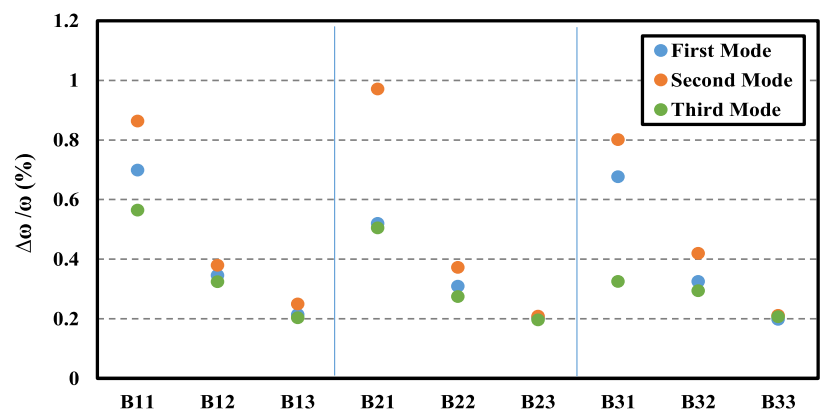

(b)

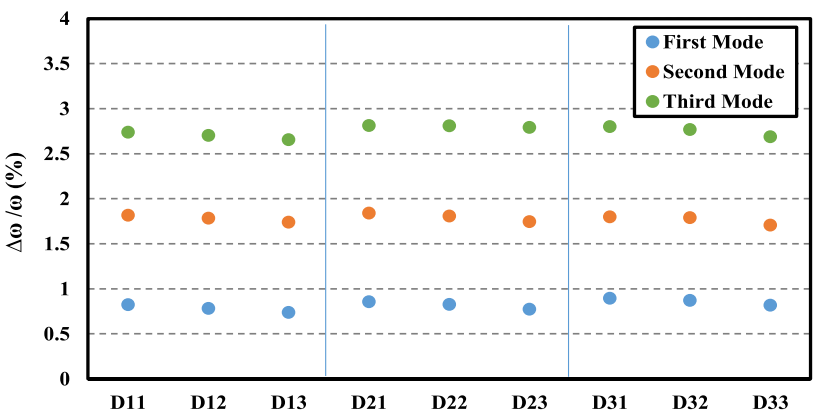

(d)

Fig. 4. Frequency sensitivity of the mass microsensor in different configurations. a: Model A; b: Model B; c: Model C; d: Model D.

the selected microbeam models in order to achieve the maximum functionality as mass sensors. Since the microbeam resonance frequency changes when the nanoparticle is placed on it, the changes in resonance frequency are a good measure for the sensor. Obviously, a greater change in the resonance frequency when a certain mass of nanoparticles in placed on the beam means that the microbeam has a better ability as a mass sensor.

Figure 4 shows the frequency sensitivity of three selected microbeam models to absorption of a nanoparticle with a mass of $0.1 \mathrm{ng}$. $\Delta \omega$ represents the change in the resonance frequency of the microbeam after the nanoparticle is absorbed by the microbeam at its tip. The results presented in Figure 4a indicate that, in the third vibrating mode, models $(\mathrm{A}),(\mathrm{C})$ and $(\mathrm{D})$ have the best frequency sensitivity to particle absorption in all selected configurations. However, the model (B) microbeam has the best frequency sensitivity in the second vibrating mode. According to Figure 4a, increasing the length of the narrow part of the microbeam model (A) and, thereby, the reduced microbeam stiffness, increases its frequency sensitivity. As evident from Figure $4 \mathrm{~b}$, the behavior of the microbeam model (B) is opposite to that of the model (A) due to its geometry. The results presented in Figures 4c and 4d indicate that increasing the length of the cut section and, thereby, increased microbeam stiffness, reduces the frequency sensitivity of the microbeam.

The general comparison of the results of Figure 4 shows that the hollow triangular microbeam (model D) has the highest sensitivity to absorbed nanoparticle mass. There- fore, this type of beam is the most suitable model for the mass sensor application among the four models studied. The figure shows that model $\mathrm{C}$ is the most unfit to be used as a mass sensor. Among the four selected models, the triangular hollow microbeam has the least sensitivity to the change in aspect ratios.

\section{The actuation capability of the microbeam}

Vibrating actuation of microbeams in microelectromechanical systems is basically done by two methods of actuation though base and self-actuation. In the actuation though base method, the supported end of the beam vibrates and stimulates the microbeam. Another method used for vibrating actuation of microbeams is the use of a piezoelectric layer on the microbeam, which is called selfactuation. In this method, the piezoelectric layer vibrated though creating an alternating potential difference at the top and bottom of the layer, inducing vibration in the microbeam. Certainly, the greater is the microbeam vibrating amplitude for the particular actuation, the greater is the microbeam actuation. This means that the microbeam needs a lower energy input to achieve the desired vibration amplitude.

In order to compare the actuation ability of the microbeam in the base actuation and the self-actuation cases, each microbeam is stimulated at the same conditions based on the aspect ratios of Tables 1-4. For the base actuation, the base amplitude was considered $2 \mathrm{~nm}$, and for the self-actuation case, the piezoelectric layer was consid- 


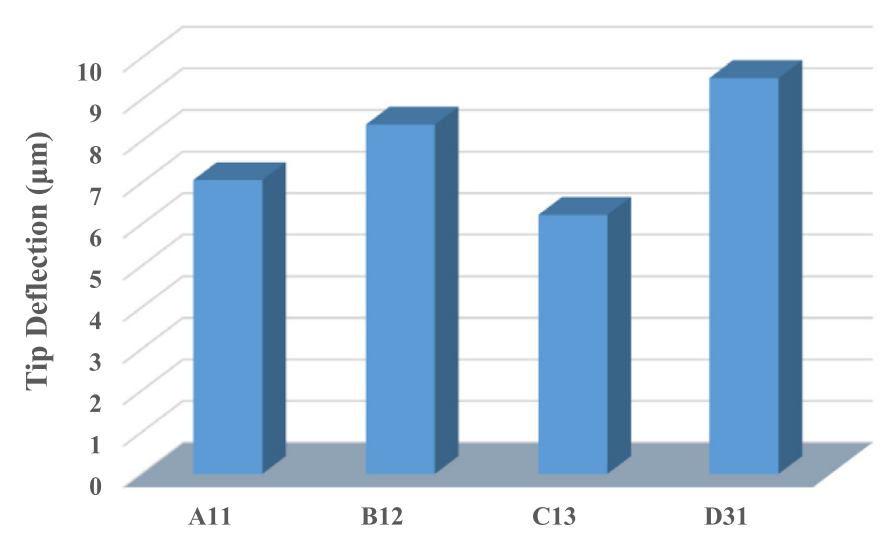

Fig. 5. Aspect ratios with the maximum tip deflection in the actuation of the base case.

ered on the microbeam. The thickness of the piezoelectric layer is assumed to be equal to the thickness of the main layer. A potential difference of 10 volts is applied on both sides of the piezoelectric layer. In all cases, actuation occurs at the microbeam resonance frequency. In these analyzes, the length, thickness, and width of the microbeam at the support are assumed to be the same in all cases. Figures 5 and 6 show the configuration of each model, in which the vibrating amplitude reaches its maximum in the actuation of the base and the self-actuation cases, respectively. The results of Figures 4 and 5 show aspect ratios with the maximum tip deflection in same actuation.

\section{Conclusion}

Microbeams are widely used as sensors and actuators in nanotechnology, biology, and optics. Given their microscale dimensions, they have great potential as a nanoscale mass sensor and even at smaller scales. In sensors and particularly mass sensors, a challenge is to enhance the sensitivity and, thereby, the accuracy. The geometric shape of the microbeam is an important parameter affecting the sensitivity of these sensors. In this paper, rectangular and triangular microbeams, which are the most commonly used mass sensors, were investigated. Four most popular models were selected by applying inner and outer cuts on the rectangular and triangular microbeams, and a total of thirty-six mass sensor configurations were simulated using ABAQUS software with different aspect ratios.

Simulation results for the 36 different selected microbeam configurations with different aspect ratios show that: - increasing the length of the narrow part of the microbeam model (A) and, thereby, the reduced microbeam stiffness, decreases the resonance frequency in the first three modes;

- in the third vibrating mode, and in all studied configurations, models (A), (C) and (D) had the best frequency sensitivity to particle absorption; meanwhile, in the second vibrating mode, the microbeam model (B)

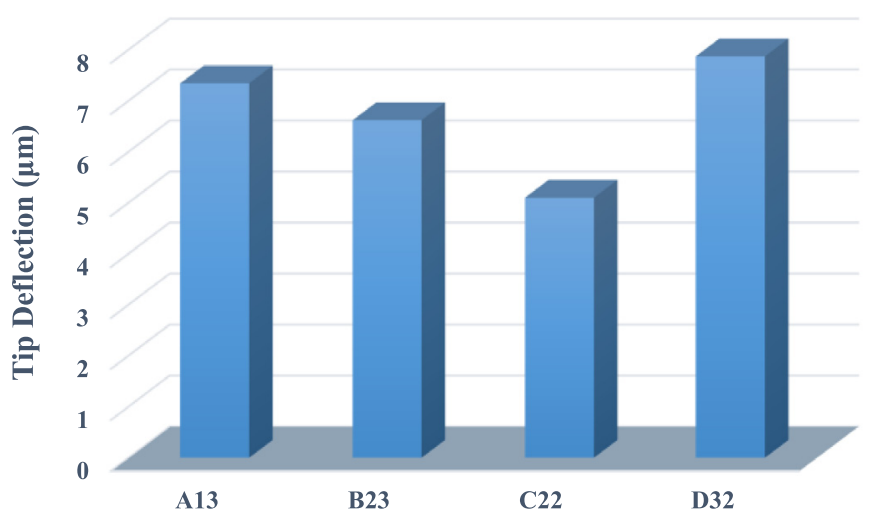

Fig. 6. Aspect ratios with the maximum tip deflection in piezoelectric actuation case.

had the best frequency sensitivity. The modes in which the microbeam has the highest frequency sensitivity are the best for the mass sensor application;

- the frequency sensitivity of the microbeam increased with the rigidity in every microbeam model studied;

- the hollow triangular microbeams (model D) had the highest mass sensitivity as mass sensors. The model $\mathrm{C}$ was also the most unfit model to be used as a mass sensor; - among the four selected models, the triangular hollow microbeam was the least sensitive to changes in aspect ratio.

\section{References}

[1] R.K. Gupta, Q. Shi, L. Dhakar, T. Wang, C.H. Heng, Ch. Lee, Broadband energy harvester using non-linear polymer spring and electromagnetic/triboelectric hybrid mechanism, Sci. Rep. 7 (2017) 41396

[2] J. Liu, L. Yuan, J. Lei, W. Zhu, B. Cheng, Q. Zhang, Y. Song, Ch. Chen, H. Xiao, Micro-cantilever-based fiber optic hydrophone fabricated by a femtosecond laser, Opt. Lett. 42 (2017) 2459-2462

[3] A.E. Ahmed, L. Trabzon, A stepwise approach for piezoresistive microcantilever biosensor optimization, World Academy of Science, Engineering and Technology, Int. J. Biomed. Biol. Eng. 11 (2017) 603

[4] L.T. Mazzola, S. Fodor, Imaging biomolecule arrays by atomic force microscopy, Biophys. J. 68 (1995) 1653-1660

[5] U. Dammer, M. Hegner, D. Anselmetti, P. Wagner, M. Dreier, W. Huber, H.-J. Güntherodt, Specific antigen/ antibody interactions measured by force microscopy, Biophys. J. 70 (1996) 2437-2441

[6] S. Adhikari, Inertial mass sensing with low Q-factor vibrating microcantilevers, J. Appl. Phys. 122 (2017) 144304

[7] M. Okan, M. Duman, Functional polymeric nanoparticle decorated microcantilever sensor for specific detection of erythromycin, Sens. Actuators B: Chem. 256 (2018) 325-333

[8] M. Alvarez, J. Plaza, L. Villanueva, C. Dominguez, L. Lechuga, Asymmetrically coupled resonators for mass sensing, Appl. Phys. Lett. 111 (2017) 113101 
[9] J.H. Lee, K.H. Yoon, K.S. Hwang, J. Park, S. Ahn, T.S. Kim, Label free novel electrical detection using micromachined PZT monolithic thin film cantilever for the detection of C-reactive protein, Biosens. Bioelectron. 20 (2004) 269-275

[10] P.M. Kosaka, V. Pini, M. Calleja, J. Tamayo, Ultrasensitive detection of HIV-1 p24 antigen by a hybrid nanomechanical-optoplasmonic platform with potential for detecting HIV-1 at first week after infection, PloS One 12 (2017) e0171899

[11] F.T. Goericke, W.P. King, Modeling piezoresistive microcantilever sensor response to surface stress for biochemical sensors, IEEE Sens. J. 8 (2008) 1404-1410

[12] A. Loui, F. Goericke, T. Ratto, J. Lee, B. Hart, W. King, The effect of piezoresistive microcantilever geometry on cantilever sensitivity during surface stress chemical sensing, Sens. Actuators A: Phys. 147 (2008) 516-521

[13] A. Salehi-Khojin, S. Bashash, N. Jalili, M. Müller, R. Berger, Nanomechanical cantilever active probes for ultrasmall mass detection, J. Appl. Phys. 105 (2009) 013506

[14] H. Xie, J. Vitard, S. Haliyo, S. Régnier, Enhanced sensitivity of mass detection using the first torsional mode of microcantilevers, Meas. Sci. Technol. 19 (2008) 055207

[15] S. Faegh, N. Jalili, O. Yavuzcetin, D. Nagesha, R. Kumar, S. Sridhar, A cost-effective self-sensing biosensor for detection of biological species at ultralow concentrations, J. Appl. Phys. 113 (2013) 224905

[16] S. Faegh, N. Jalili, S. Sridhar, Ultrasensitive piezoelectricbased microcantilever biosensor: Theory and experiment, IEEE/ASME Trans. Mechatron. 20 (2015) 308

Cite this article as: J.R. Ardali, R. Ghaderi, F. Raeiszadeh, Effect of microbeam geometry on the nano-mass sensor performance, Mechanics \& Industry 20, 304 (2019) 